\title{
Effects of ERK1/2 S-nitrosylation on ERK1/2 phosphorylation and cell survival in glioma cells
}

\author{
LEI JIN ${ }^{1,2^{*}}$, YUJIA CAO ${ }^{3 *}$, TONG ZHANG ${ }^{1,2^{*}}$, PENG WANG $^{1,2}$, DAOFEI JI ${ }^{1,2}$, XUEJIAO LIU $^{1,2}$, \\ HENGLIANG SHI $^{1,2}$, LEI HUA ${ }^{2}$, RUTONG YU ${ }^{1,2}$ and SHANGFENG GAO ${ }^{1,2}$ \\ ${ }^{1}$ Institute of Nervous System Diseases, Xuzhou Medical University; ${ }^{2}$ Department of Neurosurgery, Brain Hospital, \\ Affiliated Hospital of Xuzhou Medical University, Xuzhou, Jiangsu 221002; ${ }^{3}$ Department of Neurosurgery, \\ People's Hospital of Gaoxin District, Suzhou, Jiangsu 215011, P.R. China
}

Received December 7, 2016; Accepted December 1, 2017

DOI: $10.3892 /$ ijmm.2017.3334

\begin{abstract}
Aberrant activation of extracellular signal-regulated kinase $1 / 2($ ERK1/2) by phosphorylation modification can trigger tumorcell development in glioma. S-nitrosylation, which refers to the covalent addition of a nitric oxide (NO) group to a cysteine (Cys) thiol, is an important post-translational modification that occurs on numerous cancer-associated proteins. Protein S-nitrosylation can increase or decrease protein activity and stability, and subsequent signal transduction and cellular processes. However, the association between ERK1/2 S-nitrosylation and ERK1/2 phosphorylation, and the effects of ERK1 S-nitrosylation on glioma cell survival are currently unknown. U251 glioma cells were treated with NO donors sodium nitroprusside (SNP) or S-nitrosoglutathione (GSNO). CCK8 assay was used to assess the cell viability. NO levels in the medium were detected by Griess assay. Western blot analysis and biotin switch assay were employed to detect the ERK1/2 phosphorylation and S-nitrosylation. ERK1 wild-type and mutant plasmids were constructed, and used to transfect the U251 cells. Caspase-3 western blot analysis and flow cytometry were employed to assess cell apoptosis. The present study demonstrated that treatment with the NO donors SNP or GSNO led to an increase in ERK1/2 S-nitrosylation, and a reduction in ERK1/2 phosphorylation, which was accompanied by growth inhibition of U251 glioma cells. Mutational analysis demonstrated that $\mathrm{Cys}^{183}$ was vital for S-nitrosylation of ERK1, and that preventing ERK1 S-nitrosylation by replacing Cys ${ }^{183}$ with alanine partially reversed GSNO-induced cell apoptosis, and reductions in cell viability and ERK1/2 phosphorylation.
\end{abstract}

Correspondence to: Dr Shangfeng Gao or Dr Rutong Yu, Institute of Nervous System Diseases, Xuzhou Medical University, 84 West Huai-Hai Road, Xuzhou, Jiangsu 221002, P.R. China

E-mail: gaoshangfeng@xzhmu.edu.cn

E-mail: yu.rutong@163.com

*Contributed equally

Key words: nitric oxide, extracellular signal-regulated kinase 1/2, S-nitrosylation, phosphorylation, apoptosis, glioma
In addition, increased ERK1/2 phosphorylation was associated with decreased ERK1/2 S-nitrosylation in human glioma tissues. These findings identified the relationship between ERK1/2 S-nitrosylation and phosphorylation in vitro and in vivo, and revealed a novel mechanism of ERK1/2 underlying tumor cell development and apoptotic resistance of glioma.

\section{Introduction}

Glioma is the most common type of intracranial primary malignant tumor, which is associated with a poor median survival time of $<15$ months (1). Existing therapies include surgical removal, chemotherapy and radiotherapy; however, they are often unsuccessful (1-3). The difficulties in curing glioma are due to uncontrollable proliferation and infiltrative growth (1), which are considered to be largely attributed to aberrant signaling (4).

Mitogen-activated protein kinase (MAPK) cascades have been widely studied and are reported to be markedly altered in glial tumors $(4,5)$. Extracellular signal-regulated kinase $1 / 2(E R K 1 / 2)$ is a crucial member of the MAPK family, which contains a conserved and dual-specificity motif (T-E-Y) that can be phosphorylated on threonine (Thr) ${ }^{202}$ and tyrosine $(\mathrm{Tyr})^{204}$ residues. ERK1/2 is involved in the regulation of cell cycle progression, proliferation, differentiation, senescence and apoptosis (6). In human glioma tissues, the expression levels of phosphorylated (p)-ERK1/2 are significantly increased compared with in normal brain tissues, and expression is correlated with glioma grade $(7,8)$, thus suggesting that aberrant upregulation or activation of ERK1/2 may lead to malignant progression of glioma. However, pharmacological inhibitors of ERK1/2 are cytostatic at best, and only in a subset of patients (4), thus indicating that other unidentified factors or compensatory signals may affect the survival and growth of tumor cells.

Nitric oxide (NO) is a short-lived free radical, which serves critical roles in the regulation of cardiovascular, immune and central nervous systems (9). S-nitrosylation refers to the covalent addition of a NO group to a cysteine (Cys) thiol, and is considered one of the important ways through which NO functions (10). Protein S-nitrosylation can alter spatial structure of proteins, and increase or decrease protein activity and stability and subsequent signal transduction and cellular processes (11). 
Feng et al reported that ERK1 harbors six Cys residues and that $\mathrm{Cys}^{183}$ is the key site for ERK1 nitrosylation (12). The present study aimed to investigate the association between ERK1/2 nitrosylation and ERK1/2 phosphorylation, and the effects of ERK1 S-nitrosylation at Cys ${ }^{183}$ on glioma cell survival.

The results of the present study demonstrated that treatment with the NO donors sodium nitroprusside (SNP) or S-nitrosoglutathione (GSNO) induced an increase in ERK1/2 S-nitrosylation, and a reduction in ERK1/2 phosphorylation, which were accompanied by growth inhibition of U251 glioma cells. Mutational analysis [Cys ${ }^{183}$ to alanine (Ala $\left.{ }^{183}\right]$ uncovered that S-nitrosylation of ERK1 attenuated ERK1/2 phosphorylation, inhibited cell survival and promoted apoptosis. In addition, the results detected an increase in phosphorylation of ERK1/2 and a decrease in ERK1/2 S-nitrosylation in human glioma tissues. These findings identified a novel mechanism of ERK1/2 underlying tumor cell development and apoptotic resistance in glioma.

\section{Materials and methods}

Reagents and antibodies. Methyl methylthiomethyl sulfoxide (MMTS), neocuproine, sodium ascorbate and GSNO were purchased from Sigma-Aldrich (Merck KGaA, Darmstadt, Germany). SNP was obtained from Beyotime Institute of Biotechnology (Haimen, China). PolyJet ${ }^{\mathrm{TM}}$ and Biotin-HPDP were purchased from Thermo Fisher Scientific, Inc. (Waltham, MA, USA). Antibodies against Flag (F1084; 1:1,000; Sigma-Aldrich; Merck KGaA), ERK1/2 (ab17942; 1:1,000; Abcam, Cambridge, UK), p-ERK1/2 (sc-81492; 1:1,000; Santa Cruz Biotechnology, Inc., Dallas, TX, USA), and caspase-3 (GTX110543; 1:1,000; GeneTex, Inc., Irvine, CA, USA) were commercially available.

Cell culture. The U251 glioma cell line was purchased from Shanghai Cell Bank, Type Culture Collection Committee, Chinese Academy of Sciences (Shanghai, China). The cells were cultured in Dulbecco's modified Eagle's medium supplemented with $10 \%$ fetal bovine serum (HyClone; GE Healthcare Life Sciences, Logan, UT, USA) in a cell incubator containing $5 \% \mathrm{CO}_{2}$ under saturated humidity at $37^{\circ} \mathrm{C}$. Cells were treated at $37^{\circ} \mathrm{C}$ with NO donors SNP $(0-2 \mu \mathrm{M})$ or GSNO $(0-500 \mu \mathrm{M})$ for $36 \mathrm{~h}$ or with SNP $(2 \mu \mathrm{M})$ or GSNO $(500 \mu \mathrm{M})$ for different time ( $0-36$ or $48 \mathrm{~h})$ prior to further analysis.

Cell viability detection. Cell viability was measured using Cell Counting kit-8 (CCK-8; Dojindo Molecular Technologies, Inc., Kumamoto, Japan). A single cell suspension $\left(5 \times 10^{3} / \mathrm{ml}, 100 \mu \mathrm{l}\right)$ was seeded into a 96-well plate. Subsequently, $10 \mu \mathrm{l} \mathrm{CCK-8}$ reagent was added to each well and the plates were incubated for $2 \mathrm{~h}$ at $37^{\circ} \mathrm{C}$. Finally, the absorbance was measured at $450 \mathrm{~nm}$ using a scanning microplate reader. Cell viability at individual time-points was normalized to the untreated group.

Flow cytometric apoptotic assay. Cells were harvested and washed twice with ice-cold PBS, after which they were resuspended in $1 \mathrm{X}$ binding buffer [0.01 M HEPES/NaOH (pH 7.4), $0.14 \mathrm{M} \mathrm{NaCl}, 2.5 \mathrm{mM} \mathrm{CaCl}_{2}$ ] at a concentration of $10^{6}$ cells $/ \mathrm{ml}$. Subsequently, $100 \mu \mathrm{l}$ solution was transferred to a $5 \mathrm{ml}$ cell culture tube and was treated with fluorescein isothiocyanate-conjugated Annexin $\mathrm{V}$ apoptosis detection kit I (BD Biosciences, Franklin Lakes, NJ, USA) according to the manufacturer's protocol. The cells were analyzed using flow cytometry (DiVa 8.0.1; BD Biosciences) and a total of 10,000 cells/sample were analyzed to determine the percentage of apoptotic cells.

Western blot analysis. Total protein was extracted from the cells or tissues, as described previously (13). Protein concentrations were determined by a BCA protein assay kit (Beyotime Institute of Biotechnology) according to the manufacturer's instructions. Equal amounts of protein $(30 \mu \mathrm{g})$ were mixed with SDS sample buffer, separated by $10 \%$ SDS-PAGE and transferred to polyvinylidene fluoride membranes (EMD Millipore, Billerica, MA, USA). The membranes were then incubated with 3\% bovine serum albumin (Sigma-Aldrich; Merck $\mathrm{KGaA}$ ) in PBS at room temperature for $2 \mathrm{~h}$, and were treated with primary antibodies overnight at $4^{\circ} \mathrm{C}$. $\beta$-actin (sc-47778; 1:1,000; Santa Cruz Biotechnology, Inc.) was used as a protein-loading control. The next day, membranes were incubated with horseradish peroxidase-conjugated goat anti-rabbit (31460)/mouse (31430) immunoglobulin G (1:4,000; Invitrogen; Thermo Fisher Scientific, Inc.) at room temperature for $2 \mathrm{~h}$ and were then detected using a standard chemiluminescence detection system (Pierce; Thermo Fisher Scientific, Inc.). Band densities were analyzed using Image J software (Image J 1.43u; National Institute of Health, Bethesda, MD, USA).

NO detection. NO levels were determined by Griess assay using a commercial kit (Nanjing Jiancheng Bioengineering Institute, Nanjing, China). Cell supernatants $(100 \mu \mathrm{l})$ were thoroughly mixed with reagent I $(200 \mu \mathrm{l})$. The supernatants were obtained simply by a $200 \mu \mathrm{l}$ pipette from the cell medium. Subsequently, reagent II $(100 \mu \mathrm{l})$ was added and mixed for $10 \mathrm{~min}$ at room temperature, followed by centrifugation at $1,700 \mathrm{x} g$ for $15 \mathrm{~min}$ at room temperature. Finally, $160 \mu 1$ supernatants were mixed with $80 \mu \mathrm{l}$ chromogenic reagent for $15 \mathrm{~min}$ at room temperature. The optical density of the samples was measured using a spectrophotometer with absorbance set at $550 \mathrm{~nm}$. Sodium nitrite was used as a standard.

Biotin switch assay. Samples were lysed in non-denaturing lysis buffer (25 mM HEPES, $50 \mathrm{mM} \mathrm{NaCl}, 0.1 \mathrm{mM}$ EDTA, $1 \%$ NP-40 and $1 \mathrm{X}$ protease inhibitor cocktail, $\mathrm{pH}$ 7.4). Protein concentration was determined using the bicinchoninic acid protein assay kit (Beyotime Institute of Biotechnology). Protein lysates $(2 \mathrm{mg}$ ) were diluted to a final volume of $1.8 \mathrm{ml}$ using HEN buffer (100 mM HEPES, $1 \mathrm{mM}$ EDTA and $0.1 \mathrm{mM}$ neocuproine). Subsequently, $0.2 \mathrm{ml} 25 \%$ SDS and $20 \mu 110 \%$ MMTS were added to block free thiols. After removing excess MMTS by acetone precipitation, the S-nitrosothiol (SNO) groups in the samples were reduced to thiols by $30 \mu 1$ sodium ascorbate $(200 \mathrm{mM})$ and biotinylated by $30 \mu 1$ Biotin-HPDP $(2.5 \mathrm{mg} / \mathrm{ml})$. Finally, the biotinylated proteins were pulled down by streptavidin-agarose beads, and the beads were eluted by SDS loading buffer and subjected to western blot analysis.

Plasmids. Full-length wild-type ERK1 (ERK1 ${ }^{\mathrm{wT}}$ ) cDNA clone was purchased from Sino Biological, Inc. (Beijing, China) and 
Table I. Clinicopathological characteristics of the studied subjects.

\begin{tabular}{|c|c|c|c|c|c|}
\hline Case no. & Code no. & Gender & Age (years) & Location & WHO grade \\
\hline 1 & 919616 & M & 57 & Right cerebellum & Noncancerous \\
\hline 2 & 912226 & $\mathrm{~F}$ & 54 & Right temporal lobe & Noncancerous \\
\hline 3 & 972078 & $\mathrm{~F}$ & 49 & Not available & Noncancerous \\
\hline 4 & 968605 & $\mathrm{~F}$ & 69 & Not available & Noncancerous \\
\hline 5 & 981488 & M & 41 & Cerebellum & Noncancerous \\
\hline 6 & 1095392 & M & 32 & Not available & Noncancerous \\
\hline 7 & 1004728 & M & 63 & Right frontal lobe & Noncancerous \\
\hline 8 & 941814 & M & 20 & Cerebellum & Noncancerous \\
\hline 9 & 928412 & M & 48 & Not available & Noncancerous \\
\hline 10 & 970570 & M & 52 & Right frontal lobe & Grade II \\
\hline 11 & 1157139 & M & 42 & Right frontal lobe & Grade II \\
\hline 12 & 1145933 & $\mathrm{~F}$ & 48 & Right frontal lobe & Grade II \\
\hline 13 & 1140811 & $\mathrm{~F}$ & 49 & Right frontal lobe & Grade II \\
\hline 14 & 1164493 & M & 64 & Left insular lobe & Grade II \\
\hline 15 & 1190502 & M & 31 & Left frontal lobe & Grade II \\
\hline 16 & 1158620 & M & 43 & Right temporal lobe & Grade II \\
\hline 17 & 1196273 & M & 40 & Left frontal lobe & Grade II \\
\hline 18 & 1152968 & $\mathrm{~F}$ & 63 & Left temporal lobe & Grade II \\
\hline 19 & 1110685 & $\mathrm{~F}$ & 27 & Right frontal lobe & Grade II \\
\hline 20 & 1084447 & $\mathrm{~F}$ & 52 & Not available & Grade II \\
\hline 21 & 999737 & M & 52 & Left frontal-temporal lobe & Grade III \\
\hline 22 & 920498 & M & 50 & Right temporal-parietal lobe & Grade III \\
\hline 23 & 926714 & M & 56 & Bilateral temporal lobe & Grade III \\
\hline 24 & 1164248 & $\mathrm{~F}$ & 66 & Left frontal lobe & Grade III \\
\hline 25 & 1191197 & M & 68 & Left parietal-occipital lobe & Grade III \\
\hline 26 & 922050 & $\mathrm{~F}$ & 19 & Right frontal lobe & Grade III \\
\hline 27 & 1117547 & M & 47 & Right temporal lobe & Grade III \\
\hline 28 & 1081283 & $\mathrm{~F}$ & 58 & Left temporal lobe & Grade III \\
\hline 29 & 947804 & $\mathrm{~F}$ & 66 & Left frontal-temporal-parietal lobe & Grade III \\
\hline 30 & 1145935 & M & 23 & Cervical cord & Grade III \\
\hline 31 & 1029589 & M & 31 & Right frontal-temporal lobe & Grade III \\
\hline 32 & 1147279 & $\mathrm{~F}$ & 64 & Cerebellum & Grade IV \\
\hline 33 & 1147166 & M & 58 & Left frontal-temporal lobe & Grade IV \\
\hline 34 & 1141904 & M & 47 & Right temporal lobe & Grade IV \\
\hline 35 & 1132842 & $\mathrm{~F}$ & 62 & Left temporal lobe & Grade IV \\
\hline 36 & 1119597 & $\mathrm{~F}$ & 50 & Right frontal lobe & Grade IV \\
\hline 37 & 1096129 & M & 61 & Left temporal lobe & Grade IV \\
\hline 38 & 1077922 & M & 43 & Left temporal lobe & Grade IV \\
\hline 39 & 1140776 & M & 26 & Right parietal-occipital lobe & Grade IV \\
\hline 40 & 1088070 & $\mathrm{~F}$ & 58 & Right temporal lobe & Grade IV \\
\hline 41 & 1164493 & M & 66 & Left insular lobe & Grade IV \\
\hline 42 & 1184604 & $\mathrm{~F}$ & 34 & Right temporal lobe & Grade IV \\
\hline
\end{tabular}

F, female; M, male; WHO, World Health Organization.

was subcloned into the $3 x$ Flag vector. The primer sequences used for construction of Flag-ERK $1^{\text {WT }}$ were as follows: Forward, 5'-CCGGAATTCATGGCGGCGGCGGCGGC TCA-3' and reverse, 5'-CGCGGATCCGGGGGCCTCCAG CACTCCGG-3'. C183A mutant ERK1 (ERK1 ${ }^{\mathrm{C1} 183 \mathrm{~A}}$ ) cDNA was obtained by polymerase chain reaction [PCR; Tiangen Biotech
(Beijing) Co., Ltd., Beijing, China] with the Flag-ERK1 ${ }^{\text {WT }}$ plasmid used as the template, and was then subcloned into the $3 x$ Flag vector. The primer sequences used were as follows: forward, 5'-CCTTAAGATTGCTGATTTCGGCCTGGC-3' and reverse, 5'-GCCGAAATCAGCAATCTTAAGGTC GCAG-3'. PCR thermocycling was performed as follows: $94^{\circ} \mathrm{C}$ 

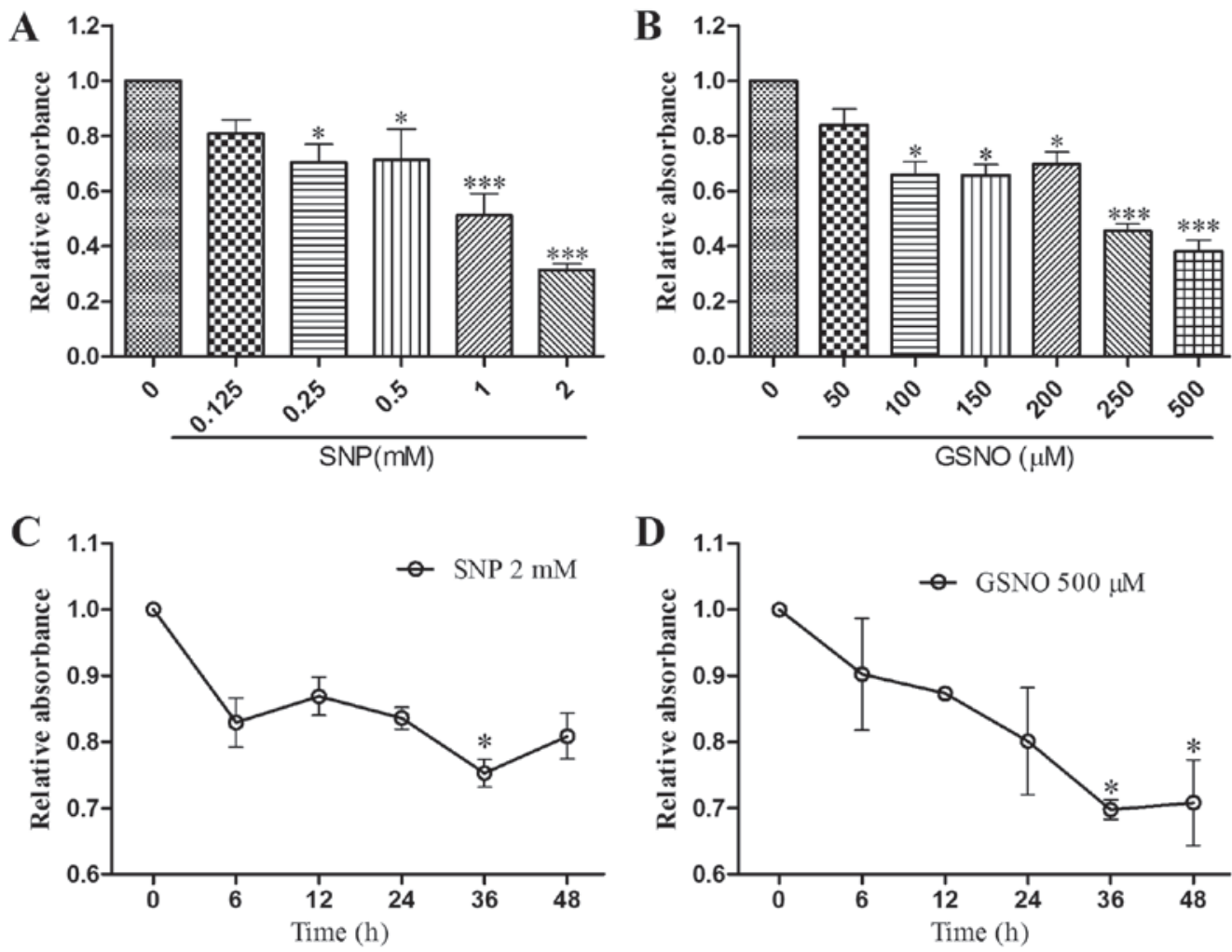

Figure 1. NO donor treatment inhibits the growth of U251 glioma cells. (A and B) Results of a CCK-8 assay indicated a concentration-dependent decrease in the viability of U251 glioma cells following exposure to 0-2 mM SNP or 0-500 $\mu \mathrm{M}$ GSNO for $36 \mathrm{~h}$. (C and D) CCK-8 assay exhibited a reduction in cell viability at different time-points following SNP $(2 \mathrm{mM})$ or GSNO $(500 \mu \mathrm{M})$ treatment. Relative absorbance was normalized to the untreated group $(0)$. "P $<0.05$ and ${ }^{* * * *} \mathrm{P}<0.001$ compared with the untreated group (0). CCK-8, Cell Counting kit-8; GSNO, S-nitrosoglutathione; NO, nitric oxide; SNP, sodium nitroprusside.

for $3 \mathrm{~min}$; followed by 35 cycles at $90^{\circ} \mathrm{C}$ for $30 \mathrm{sec}, 60^{\circ} \mathrm{C}$ for $45 \mathrm{sec}$ and $72^{\circ} \mathrm{C}$ for $90 \mathrm{sec} ; 72^{\circ} \mathrm{C}$ for $10 \mathrm{~min}$; hold at $4^{\circ} \mathrm{C}$. The authenticity of the plasmids was confirmed by DNA sequencing. Briefly, when U251 cells reached a confluency of $40-50 \%$, the cell medium was replaced with $2 \mathrm{ml}$ fresh DMEM medium at $30 \mathrm{~min}$ before transfection. Plasmid $(1 \mu \mathrm{g})$ and PolyJet $^{\mathrm{TM}}$ reagent $(3 \mu \mathrm{l})$ were mixed in high glucose DMEM medium at room temperature. The mixture was evenly added into the medium and the cells were incubated at $37^{\circ} \mathrm{C}$ for $6-8 \mathrm{~h}$ before replacing the medium with $2 \mathrm{ml}$ fresh medium. After $48 \mathrm{~h}$, the cells were used for the experiments. Transient transfection was performed using PolyJet ${ }^{\mathrm{TM}}$ according to the manufacturer's protocol. Expression of proteins was verified by western blot analysis using ERK1/2 and Flag antibodies.

Glioma and noncancerous human brain tissue collection. Human glioma specimens, collected during surgical resection, and noncancerous brain tissues, collected during internal decompression after cerebral trauma, were obtained from the Affiliated Hospital of Xuzhou Medical University (Xuzhou, China). The clinicopathological characteristics of all of the subjects are presented in Table I. Surgically removed tissues were sampled for histological diagnosis, and the remaining tissues were immediately frozen and stored in liquid nitrogen for further analysis. All glioma specimens had a confirmed pathological diagnosis and were classified according to World Health Organization criteria (14). Informed consent was obtained from all subjects, or legal guardians, and the present study was approved by the Medical Ethical Committee of Xuzhou Medical University.
Statistical analysis. All quantitative data were obtained from at least three independent experiments and are presented as the means \pm standard error of the mean [SPSS version 13.0 (SPSS, Inc., Chicago, IL, USA)]. Data between two groups were assessed by Student's t-test, whereas one-way analysis of variance followed by Dunnett post hoc comparison was used to analyze data among three groups or more. $\mathrm{P}<0.05$ was considered to indicate a statistically significant difference.

\section{Results}

NO donor treatment inhibits growth of glioma cells. According to the literature, treatment with the NO donor SNP for 30-40 h significantly inhibits the growth of human glioma cells (15); therefore, the present study conducted a CCK- 8 assay to evaluate the concentration-dependent effects of SNP and GSNO on cell survival after 36 h in U251 cells. Cell viability was significantly reduced following treatment with 0.25 and $0.5 \mathrm{mM}$ SNP or 100-200 $\mu \mathrm{M}$ GSNO, and was reduced to a greater extent following treatment with 1 and $2 \mathrm{mM} \mathrm{SNP}$ or 250 and $500 \mu \mathrm{M}$ GSNO (Fig. 1A and B). To determine the appropriate duration of high-dose SNP or GSNO treatment, U251 glioma cells were treated with $2 \mathrm{mM} \mathrm{SNP}$ or $500 \mu \mathrm{M}$ GSNO for 0, 6, 12,24, 36 and $48 \mathrm{~h}$. Significant inhibition of cell survival was observed when cells were exposed to $2 \mathrm{mM} \mathrm{SNP}$ for $36 \mathrm{~h}$ or $500 \mu \mathrm{M}$ GSNO for 36 and $48 \mathrm{~h}$ (Fig. 1C and D). These results indicated that high doses of NO donors exert significant inhibitory effects on the growth of glioma cells. 

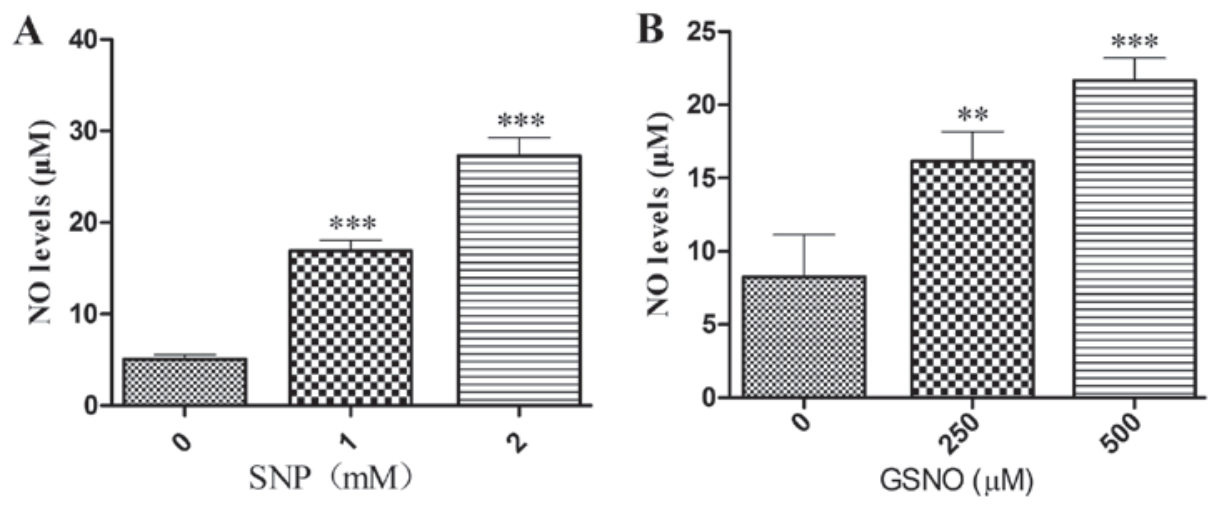

Figure 2. NO donors SNP and GSNO can release NO into the medium of cultured glioma cells. The Griess method was used to quantify NO levels in the culture supernatant of $\mathrm{U} 251$ cells following (A) 1 and $2 \mathrm{mM} \mathrm{SNP}$ or (B) 250 and $500 \mu \mathrm{M}$ GSNO treatment. ${ }^{* *} \mathrm{P}<0.01$ and ${ }^{* * *} \mathrm{P}<0.001$ compared with the untreated group (0). GSNO, S-nitrosoglutathione; NO, nitric oxide; SNP, sodium nitroprusside.

A

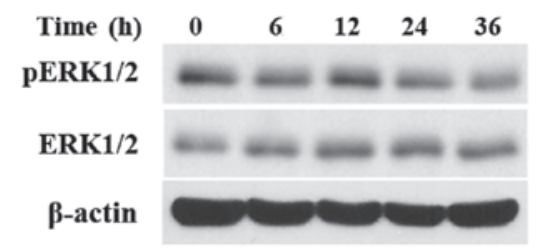

C
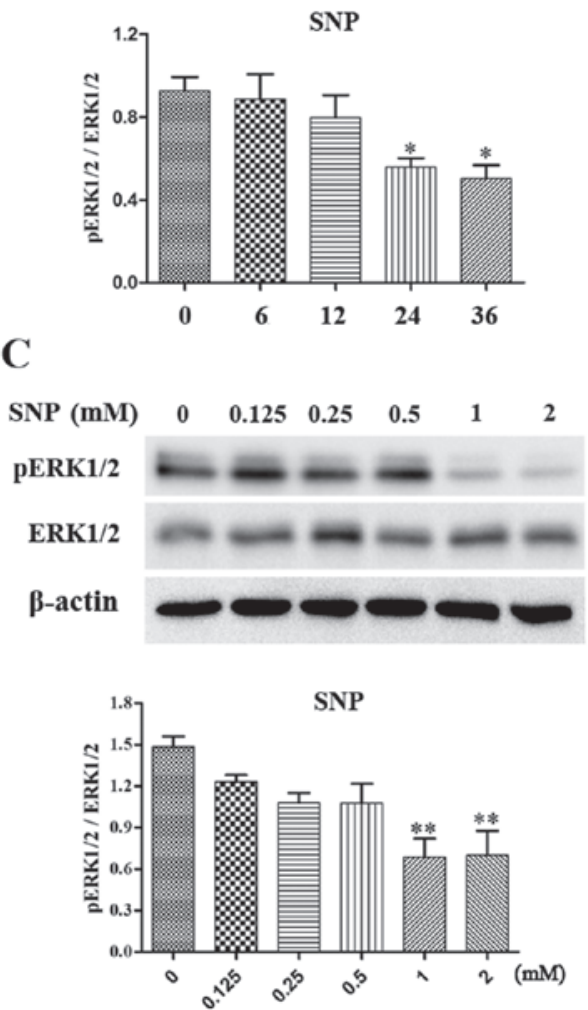

B

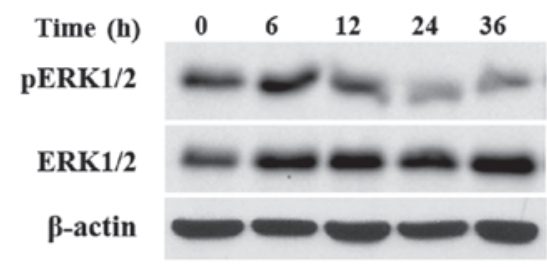

D
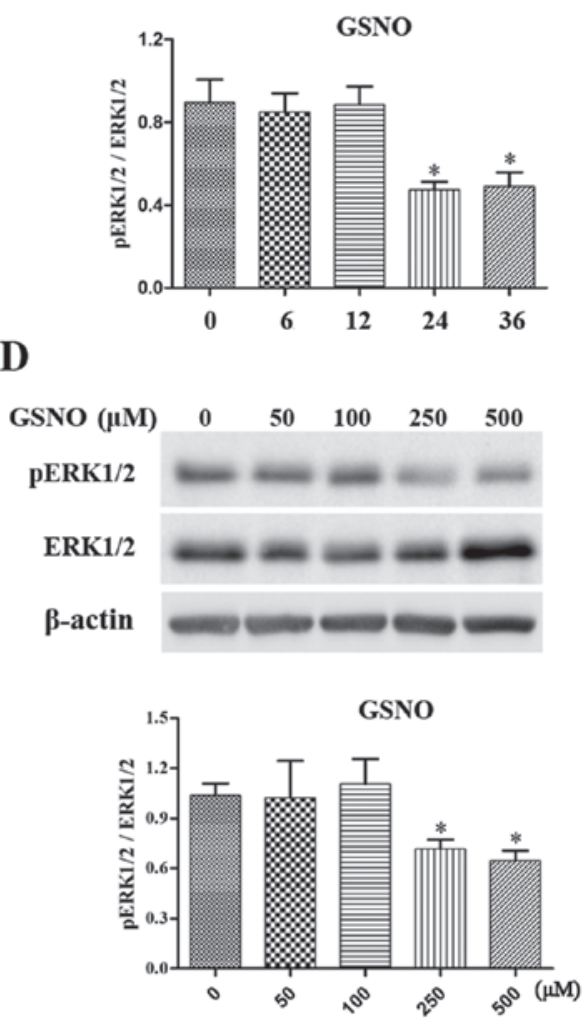

Figure 3. NO donor treatment attenuates ERK1/2 phosphorylation in U251 glioma cells. (A and B) Alterations in p-ERK1/2 expression at the indicated time-points following SNP $(2 \mathrm{mM})$ or GSNO $(500 \mu \mathrm{M})$ treatment were detected by western blotting and were semi-quantified. (C and D) Alterations in p-ERK1/2 expression following treatment with the indicated concentrations of SNP or GSNO were examined by western blotting and were semi-quantified. p-ERK1/2 expression was normalized to total ERK1/2. ${ }^{*} \mathrm{P}<0.05$ and ${ }^{* *} \mathrm{P}<0.01$ compared with the control group (0). ERK1/2, extracellular signal-regulated kinase 1/2 GSNO, S-nitrosoglutathione; NO, nitric oxide; p-ERK1/2, phosphorylated-ERK1/2; SNP, sodium nitroprusside.

NO donor treatment increases $N O$ release into the cell supernatant. To ascertain whether the NO donors SNP and GSNO could release NO, the Griess method was used to measure NO levels in the supernatant of cultured U251 cells. Significant increases in NO release were detected following treatment with 1 or $2 \mathrm{mM} \mathrm{SNP} \mathrm{(Fig.} \mathrm{2A)} \mathrm{and} 250$ or $500 \mu \mathrm{M}$ GSNO (Fig. 2B). 

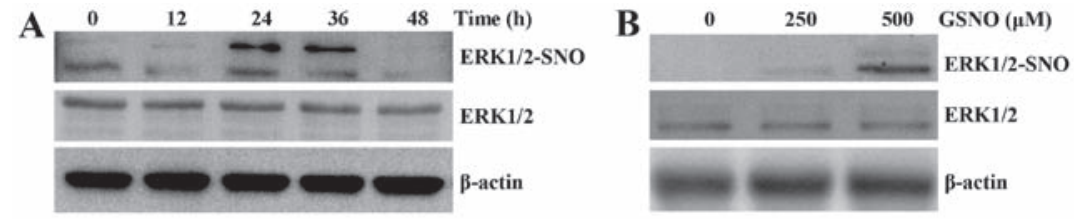

Figure 4. NO donor treatment promotes S-nitrosylation of ERK1/2 in U251 glioma cells. (A) Cells were treated with $500 \mu \mathrm{M}$ GSNO for the indicated time and ERK1/2-SNO was detected by biotin switch assay followed by western blotting. (B) Cells were treated with 250 and $500 \mu \mathrm{M}$ GSNO for $24 \mathrm{~h}$ and the level of ERK1/2-SNO was detected as aforementioned. Total ERK1/2 was used as an endogenous control. ERK1/2, extracellular signal-regulated kinase1/2; GSNO, S-nitrosoglutathione; NO, nitric oxide; SNO, S-nitrosothiol.

\section{A ERK1 Fasta \\ MAAAAAQGGGGGEPRRTEGVGPGVPGEVEMVKGQPFDVGPRYTQLQYIGE GAYGMVSSAYDHVRKTRVAIKKISPFEHQTYCQR TLREIQILLRFRHENV IGIRDILRASTLEAMRDVYIVQDLMETDLYKLLKSQQLSNDHICYFLYQI LRGLKYIHSANVLHRDLKPSNLLIN ITCDI KICDF GLARIADPEHDHTGF LTEYVATRWYRAPEIMLNSKGYTKSIDIW VGCH AEMLSNRPIFPGKHY LDQLNHILGILGSPSQEDLNCIINMARNYLQSLPSKTKVAWAKLFPKSD SKALDLLDRMLTFNPNKRITVEEALAHPYLEQYYDPTDEPVAEEPFTFAM ELDDLPKERLKELIFQETARFQPGVLEA}
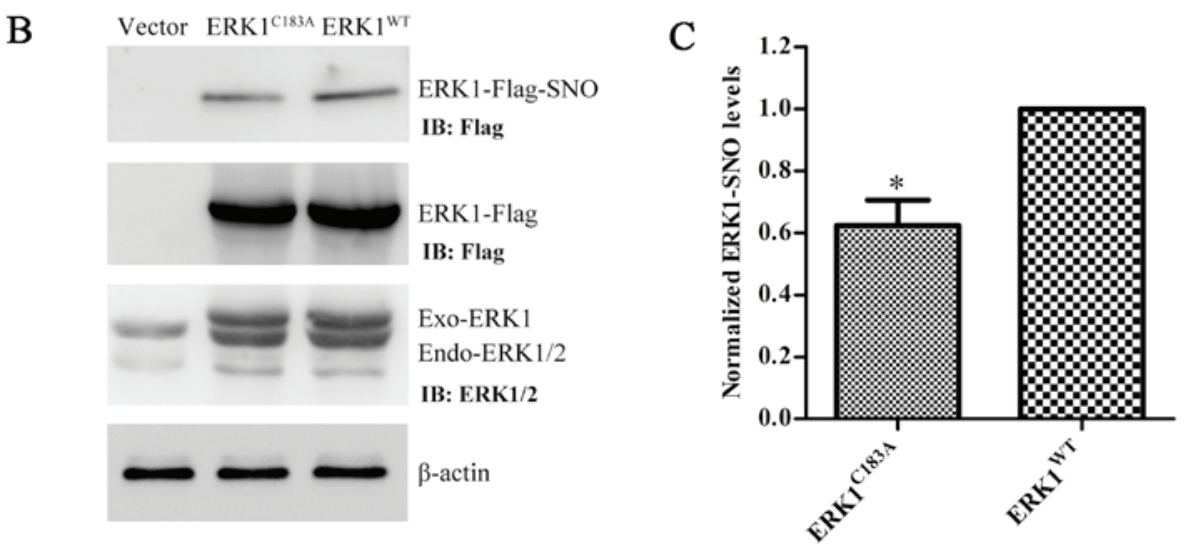

Figure 5. Point mutation at $\mathrm{Cys}^{183}$ partially prevents S-nitrosylation of ERK1 in glioma cells. (A) Location of Cys and the adjacent amino acids in the ERK1 protein sequence. The red ' $\mathrm{C}$ ' in the middle of each box is cysteine residue. (B) Transfection efficiency and S-nitrosylation of ERK1 ${ }^{\mathrm{WT}}$ and ERK1 $1^{\mathrm{C} 183 \mathrm{~A}}$ were determined by biotin switch assay, followed by western blotting. (C) Semi-quantitative analysis of ERK1-SNO levels. $\beta$-actin was used as a loading control. ${ }^{*} \mathrm{P}<0.05$ compared with the ERK1 ${ }^{\mathrm{WT}}$ group. Cys, cystein; ERK, extracellular signal-regulated kinase; SNO, S-nitrosothiol; IB, immunoblotting; WT, wild-type.

These data suggested that SNP and GSNO may breakdown to release NO into the culture supernatant of glioma cells.

NO donor treatment attenuates phosphorylation of ERK1/2 in glioma cells. The MAPK pathway has been reported to serve a critical role in cell survival (6), and increased phosphorylation of ERK1/2 has been detected in various grades of glioma $(7,8)$. The present study investigated the effects of NO donor treatment on the expression levels of p-ERK1/2 in glioma cells. Initially, a time-dependent assay was performed in U251 glioma cells. The expression levels of p-ERK1/2 were significantly reduced following treatment with $2 \mathrm{mM} \mathrm{SNP}$ or $500 \mu \mathrm{M}$ GSNO treatment for 24 and $36 \mathrm{~h}$ (Fig. 3A and B). Subsequently, U251 cells were exposed to various concentrations of SNP or GSNO for $36 \mathrm{~h}$. A concentration-dependent decrease in p-ERK1/2 expression was evident in response to SNP and GSNO treatment. Significant decreases in the expression levels of p-ERK1/2 were observed following treatment with 1 and 2 mM SNP (Fig. 3C). Similarly, p-ERK1/2 expression was significantly reduced following 250 and $500 \mu \mathrm{M}$ GSNO treatment (Fig. 3D). These data indicated that NO donor treatment, particularly in high concentrations, may attenuate the phosphorylation of ERK1/2 in glioma cells.

NO donor treatment promotes $S$-nitrosylation of ERK1/2 in glioma cells. To determine whether ERK could be nitrosylated by NO, S-nitrosylation of ERK1/2 (SNO-ERK1/2) was analyzed by a biotin switch assay, followed by western blot analysis. Time- and dose-dependent increases in the levels of SNO-ERK1/2 were detected in response to GSNO treatment of U251 cells. The levels of ERK1/2-SNO were markedly increased at 24 and $36 \mathrm{~h}$, and then returned to control levels at 48 h (Fig. 4A). ERK1/2-SNO was initially detected following treatment with $250 \mu \mathrm{M}$ GSNO and was amplified with $500 \mu \mathrm{M}$ GSNO treatment (Fig. 4B). These results suggested that ERK1/2 may be nitrosylated in a time- and dose-dependent manner.

S-nitrosylation of ERK1 is prevented by mutation at Cys ${ }^{183}$. ERK1 has six Cys residues in its FASTA sequence (Fig. 5A). A 


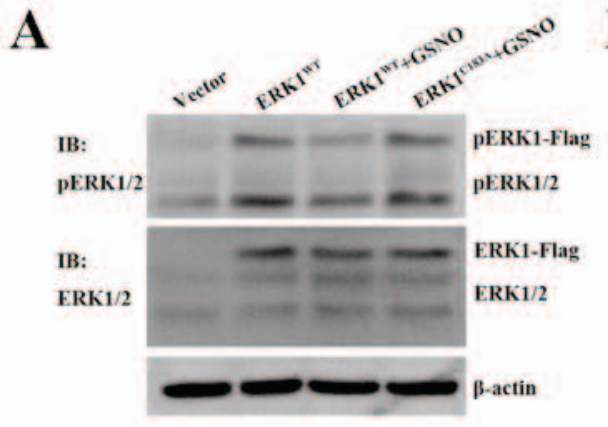

B

D

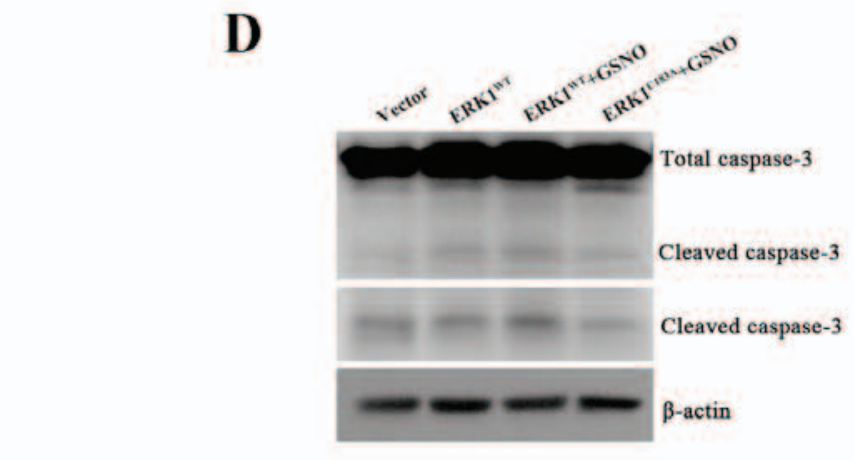

C

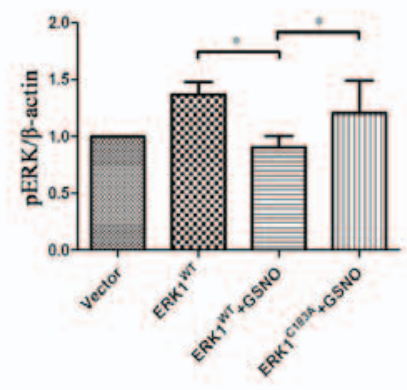

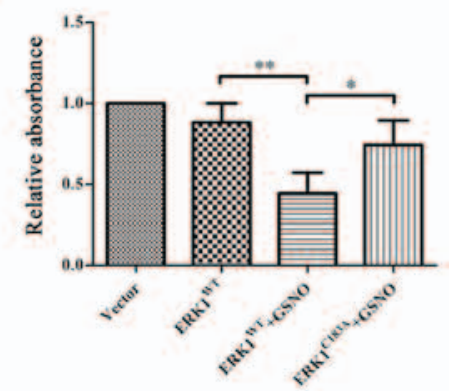

E

F

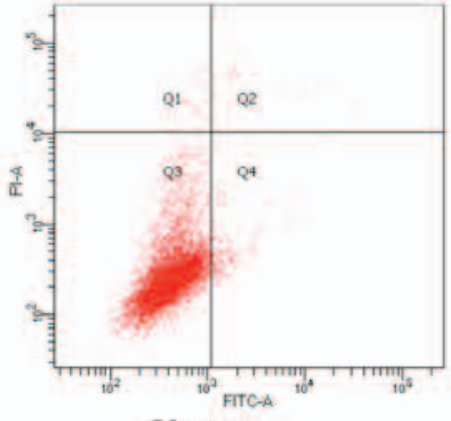

Vector

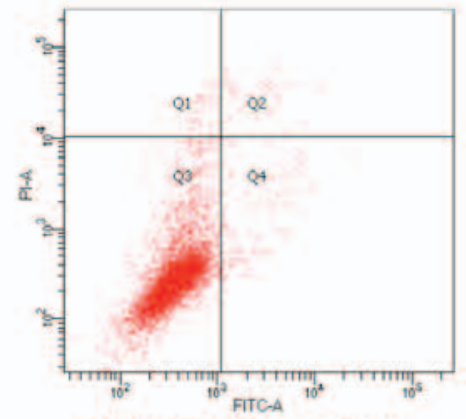

ERK1-C183A+GSNO

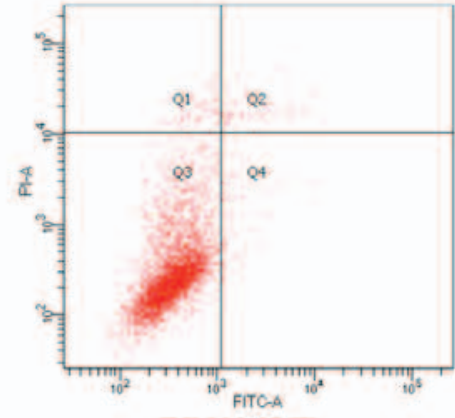

ERK1-WT

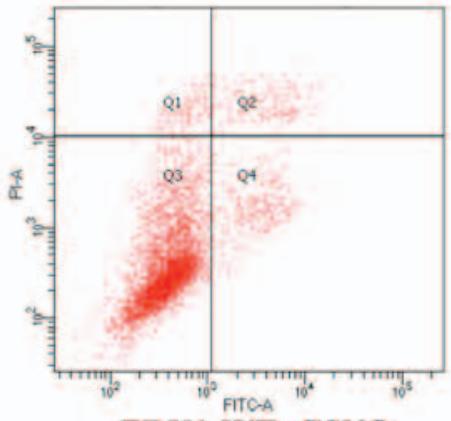

ERK1-WT+GSNO

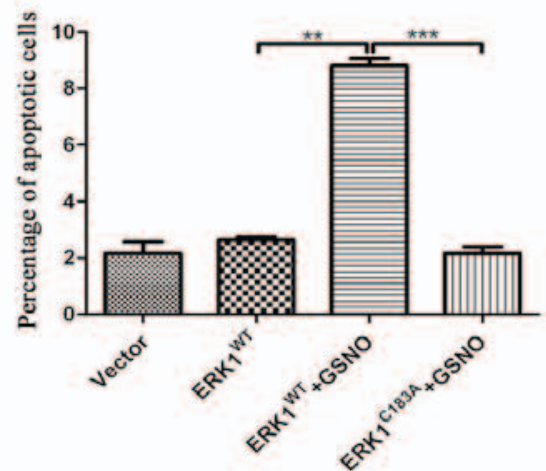

Figure 6. Preventing S-nitrosylation of ERK1 promotes ERK phosphorylation and cell survival, and suppresses apoptosis. Following transfection of U251 glioma cells with ERK1-Flag or ERK1 mutant form (ERK ${ }^{\mathrm{C} 183 \mathrm{~A}}$ ), cells were treated with $500 \mu \mathrm{M}$ GSNO for $24 \mathrm{~h}$. (A and B) p-ERK1/2 was detected by western blotting and was semi-quantified. p-ERK1/2 levels were compared with $\beta$-actin levels, and results were normalized to vector group. (C) Cell Counting kit- 8 assay was performed to examine the viability of U251 glioma cells. Cell survival percentage was normalized to the vector group. (D and E) Caspase-3 protein expression was detected by western blotting and semi-quantified. Cleaved caspase- 3 levels were compared with $\beta$-actin levels, and results were normalized to the vector group. The lower panel of cleaved caspase-3 blot in part D was obtained after a longer exposure time compared with the upper panel. (F) Flow cytometric detection of apoptosis of U251 glioma cells. The percentage of apoptotic cells was quantified and compared. ${ }^{*} \mathrm{P}<0.05,{ }^{* *} \mathrm{P}<0.01$ and ${ }^{* * * *} \mathrm{P}<0.001$. IB, immunoblotting; ERK, extracellular signal-regulated kinase; FITC, fluorescein isothiocyanate; GSNO, S-nitrosoglutathione; p-ERK1/2, phosphorylated ERK1/2; PI, propidium iodide; WT, wild-type.

preliminary computational prediction indicated that $\mathrm{Cys}^{183}$ is the most probable site for nitrosylation, according to the previ- ously reported nitrosylation motif (K/R/H/D/E+C+D/E) (16). Therefore, $\mathrm{Cys}^{183}$ was replaced with Ala, and the ERK1 mutant 

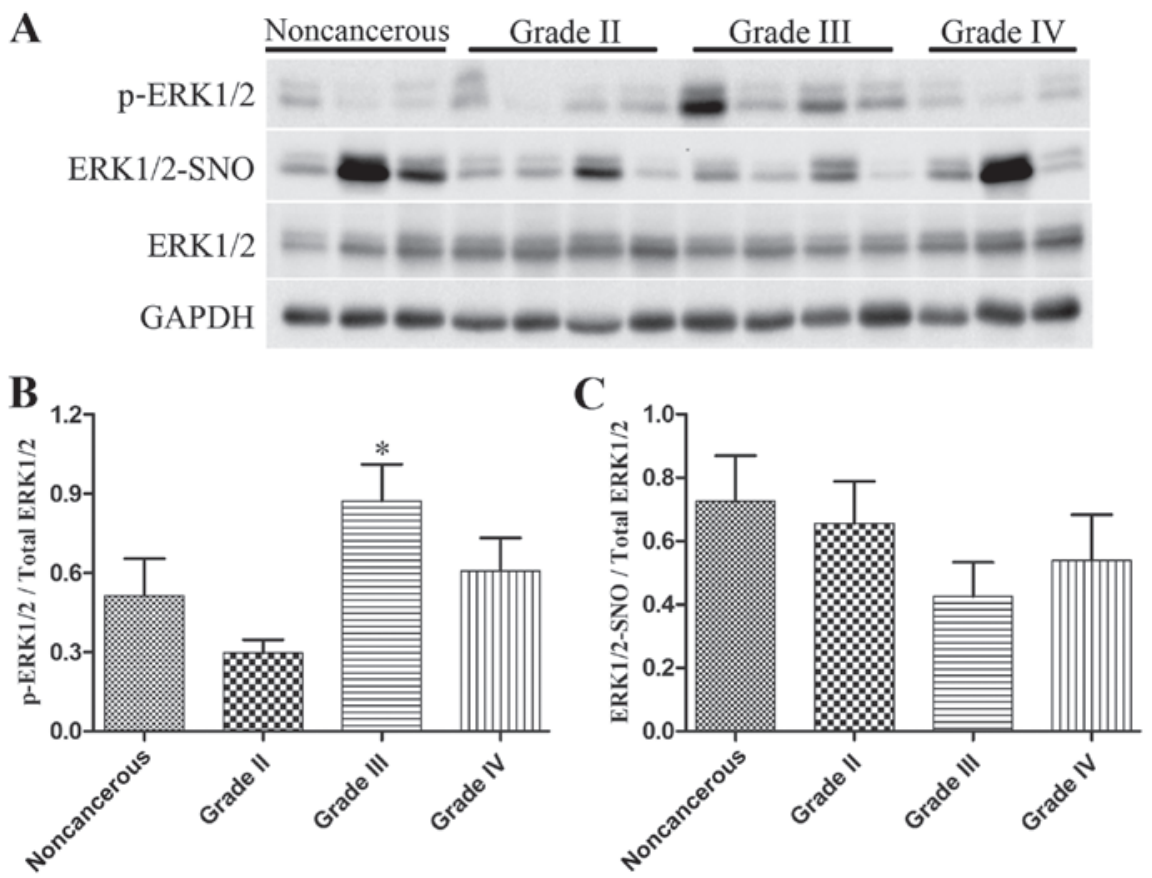

Figure 7. Alterations in the levels of ERK1/2 phosphorylation and S-nitrosylation in noncancerous and glioma tissues. In noncancerous brain samples ( $\mathrm{n}=9$ ) and various grades of glioma ( $\mathrm{n}=11$ for each grade), western blotting was used to detect the expression levels of p-ERK1/2 and total ERK1/2 levels, and biotin switch assay followed by western blotting was employed to detect ERK1/2-SNO. (A) Representative blot images are presented. Semi-quantification for the ratio of (B) p-ERK1/2/total ERK1/2 and (C) ERK1/2-SNO/total ERK1/2. ${ }^{*}<0.05$ compared with the noncancerous group. ERK1/2, extracellular signal-regulated kinase 1/2; p-ERK1/2, phosphorylated-ERK1/2; SNO, S-nitrosothiol.

plasmid (ERK1 $\left.1^{\mathrm{C} 183 \mathrm{~A}}\right)$ was constructed. Transfection efficiency of ERK $1^{\text {WT }}$ and ERK1 $1^{\mathrm{C} 183 \mathrm{~A}}$ was verified by western blot analysis using anti-Flag and anti-ERK1/2 antibodies (Fig. 5B). The expression levels of ERK1-SNO were significantly attenuated following transfection of U251 cells with the ERK1 ${ }^{\mathrm{C} 183 \mathrm{~A}}$ plasmid (Fig. 5B and C). These results suggested that mutation at $\mathrm{Cys}^{183}$ may partially prevent S-nitrosylation of ERK1/2 in glioma cells.

Preventing S-nitrosylation of ERK1 promotes ERK phosphorylation and cell survival. To determine the relationship between ERK1/2 nitrosylation and phosphorylation, U251 glioma cells were transfected with either ERK $1^{\mathrm{WT}}$ or ERK $1^{\mathrm{C} 183 \mathrm{~A}}$ plasmids, and were then treated with GSNO $(500 \mu \mathrm{M})$. The results indicated that the expression levels of p-ERK were significantly decreased in the ERK1 ${ }^{\text {WT }}$-transfected cells when GSNO was added (Fig. 6A and B), which was consistent with the previous findings presented in Fig. 3D. However, GSNO failed to reduce p-ERK1/2 levels when U251 cells were transfected with the ERK1 ${ }^{\mathrm{C} 183 \mathrm{~A}}$ mutant (Fig. 6A and B). These findings indicated that preventing ERK1 S-nitrosylation may increase the phosphorylation of ERK1/2 in glioma cells.

The present study also investigated the effects of ERK1 S-nitrosylation prevention on the survival of glioma cells. In line with the data presented in Fig. 1, cell viability was significantly reduced following treatment with GSNO; however, the reduction in cell viability was reversed by a point mutation at $\mathrm{Cys}^{183}$ of ERK1 (Fig. 6C). Furthermore, western blot analysis demonstrated that GSNO treatment induced an increase in cleaved caspase-3 expression; however, this was reversed following transfection with the ERK1 ${ }^{\mathrm{C} 183 \mathrm{~A}}$ mutant (Fig. 6D and E). In addition, flow cytometric apoptotic assay indicated that the percentage of U251 apoptotic cells transfected with ERK1 $1^{\mathrm{C} 183 \mathrm{~A}}$ mutant was significantly reduced following GSNO treatment compared with in the cells transfected with the ERK ${ }^{\mathrm{WT}}$ plasmid (Fig. 6F). Taken together, these results suggested that preventing S-nitrosylation of ERK1, via transfection with a ERK $1^{\mathrm{Cl} 183 \mathrm{~A}}$ mutant, partially reversed GSNO-induced decreases in ERK phosphorylation and cell survival in U251 glioma cells.

Alterations in ERK1/2 phosphorylation and S-nitrosylation levels in human glioma tissues. Western blot analysis was employed to detect p-ERK1/2 and total ERK1/2 levels, and a biotin switch assay followed by western blot analysis was used to measure ERK1/2-SNO levels, in 9 noncancerous brain tissues and 33 glioma specimens ( $\mathrm{n}=11 /$ grade). As presented in Fig. 7, the expression levels of $\mathrm{p}$-ERK1/2 were increased in high-grade glioma tissues, particularly in glioma grade III (Fig. 7A and B), whereas there was a marked reduction in ERK1/2-SNO levels in glioma tissues, which was also evident in glioma grade III (Fig. 7A and C). These data provided in vivo evidence for the possible influence of ERK1/2 S-nitrosylation on ERK1/2 phosphorylation during glioma proliferation.

\section{Discussion}

NO donors, SNP and GSNO, breakdown to release NO and exert an inhibitory effect on cell survival in glioma cells. In the present study, NO donor treatment induced a significant decrease in p-ERK1/2 expression (Fig. 3) and a marked increase in ERK1/2-SNO levels (Fig. 4) in U251 cells, thus suggesting a link between ERK1/2-SNO and p-ERK/2. Further mutational analysis demonstrated that $\mathrm{Cys}^{183}$ was vital for S-nitrosylation of 
ERK1 (Fig. 5) and that preventing the formation of ERK-SNO by ERK1 ${ }^{\mathrm{C} 183 \mathrm{~A}}$ mutation reversed NO-induced suppression of cell viability and p-ERK1/2 expression, and increased cell apoptosis of glioma cells (Fig. 6). In addition, increased p-ERK1/2 levels were observed in human glioma tissues, which were accompanied by a marked decrease in ERK1/2-SNO levels (Fig. 7). These findings indicated a novel mechanism underlying the antitumor role of NO-associated chemicals and provided insights into gene therapy targeting the ERK1/2 pathway in glioma.

NO is a free radical, which predominantly functions as a messenger or effector molecule. Previous studies have reported that the viability of U87 and C6cells may be significantly inhibited following exposure to high concentrations of NO donors $(15,17)$. The present study demonstrated that treatment with the NO donors SNP or GSNO resulted in a significant reduction in the viability of U251 cells. These data suggested that the inhibitory effects of NO on cell survival could be generalized in various glioma cell lines. However, previous evidence also suggests that NO displays stimulatory and inhibitory effects in the context of cell survival and apoptosis. Maejima et al reported that low doses of the NO donor S-mitroso-N-acetyl-D,L-penicillamine favor cell survival, whereas high doses may reduce cell viability of cardiomyocytes (18). Lechner et al demonstrated that low levels of NO produced by the tumor microenvironment favor tumor cell survival, whereas tumor cells with high NO levels undergo cell death (19). The dual effects of NO may be ascribed to the availability of enzymes, timing of apoptotic stimuli, redox state, donor doses, spatial location of key reactants and interactions with other molecules (20).

$\mathrm{S}$-nitrosylation is involved in the regulation of numerous biological processes, including cell proliferation and survival, and particularly apoptosis $(11,21)$. S-nitrosylation of B-cell lymphoma 2 enhances its stability, inhibits ubiquitous degradation in numerous tumor types and induces resistance to cis-platinumchemotherapy inbreastcancer $(22,23)$.Furthermore, $\mathrm{S}$-nitrosylation of the death receptor Fas initiates its redistribution on lipid rafts and promotes Fas ligand-mediated apoptosis in cancer (24). ERK1 harbors six Cys residues, as indicated in Fig. 5A. Cys ${ }^{183}$ is the most probable site for S-nitrosylation, according to the S-nitrosylation motif $(\mathrm{K} / \mathrm{R} / \mathrm{H} / \mathrm{D} / \mathrm{E}+\mathrm{C}+\mathrm{D} / \mathrm{E})$ reported previously (16). The present study indicated that ERK1/2 may be nitrosylated by the NO donor GSNO, and that replacing $\mathrm{Cys}^{183}$ with alanine may prevent the S-nitrosylation of ERK1/2 in glioma cells. The small decrease in ERK1-SNO levels in response to $\mathrm{Cys}^{183}$ mutation indicates that other ERK1 Cys residues may also contribute to S-nitrosylation. Nevertheless, the ERK $1^{\mathrm{C} 183 \mathrm{~A}}$ mutation significantly reversed GSNO-induced suppression of cell viability and enhancement of apoptosis of glioma cells. Together with a previous study in breast cancer cells (12), these findings suggested that ERK-SNO may promote tumor cell apoptosis.

Within the MAPK cascades, the ERK1/2 signaling pathway is a principle pathway that regulates cell proliferation and survival when activated by phosphorylation at $\mathrm{Thr}^{202}$ and $\mathrm{Tyr}^{204}$ residues of ERK1 (5,25). Activation of ERK1/2 signaling in glioma tissues, as determined by increased p-ERK1/2 levels, has been detected in the present study, as well as in previous studies $(7,8)$. The $\mathrm{S}$-nitrosylation site $\mathrm{Cys}^{183}$ is spatially close to $\mathrm{Thr}^{202}$ and $\mathrm{Tyr}^{204}$, thus suggesting the possibility of mutual regulation between $\mathrm{S}$-nitrosylation and phosphorylation of
ERK1/2. The present in vitro results indicated that treatment with GSNO induced a reduction in p-ERK1/2 expression, an increase in ERK1/2-SNO levels, and cell growth inhibition in glioma cells. In vivo, the results demonstrated that p-ERK1/2 levels were increased, whereas ERK1/2-SNO levels were decreased in glioma tissues, particularly in glioma grade III. Furthermore, a point mutation at $\mathrm{Cys}^{183}$ confirmed that preventing formation of ERK1-SNO significantly increased p-ERK1/2 expression and reversed GSNO-induced cell apoptosis in U251 glioma cells. These findings suggested a regulatory role of ERK1/2 S-nitrosylation on ERK1/2 phosphorylation, which may provide novel information regarding ERK1/2 targeting in glioma therapy.

In addition to ERK1/2, other important signaling proteins are also modified by S-nitrosylation. Murillo-Carretero et al reported that $\mathrm{S}$-nitrosylation of epidermal growth factor receptor (EGFR) inhibited EGFR phosphorylation and cell proliferation in neuroblastoma cells (26). In head and neck squamous cell carcinoma, S-nitrosylation of signal transducer and activator of transcription 3 (STAT3) and nuclear factor (NF) $-\kappa B$ inhibited phosphorylation of STAT3 and activation of $\mathrm{NF}-\kappa \mathrm{B}$, and decreased cell proliferation and increased apoptosis (27). These studies indicated a critical role of S-nitrosylation in the regulation of protein phosphorylation and cellular biological functions. Notably, several NO-hybridized drugs have been developed to inhibit cancer cell growth in vitro and in vivo $(28,29)$, thus suggesting the potential translational relevance of NO-mediated S-nitrosylation in the future.

A few limitations should be mentioned with regards to the present study. All in vitro work presented in this study was performed in U251 glioma cells. In this respect, duplication of efforts in other glioma cell lines would be beneficial. In addition, it is necessary to perform brain xenograft experiments to confirm the inhibitory role of ERK1 S-nitrosylation on ERK1/2 phosphorylation and glioma growth.

In conclusion, $\mathrm{NO}$ donor treatment inhibited cell survival and induced apoptosis of U251 glioma cells. S-nitrosylation of ERK1/2 and ERK1/2 phosphorylation exhibited inverse alterations in GSNO-treated glioma cells and in human glioma tissues. Preventing ERK1 nitrosylation via a mutation at $\mathrm{Cys}^{183}$ partially reversed NO-induced decreases in ERK phosphorylation and cell survival. These findings revealed a novel mechanism of ERK1/2 underlying tumor cell development and apoptotic resistance of glioma.

\section{Acknowledgements}

The present study was supported by the National Natural Science Foundation of China (grant nos. 31400930, 81472345 and 81302175), the Natural Science Foundation of Jiangsu province (grant no. BK20140217), the China Postdoctoral Science Foundation (grant nos. 2015M570480 and 2016T90505) and the Key Research and Development Plan of Jiangsu Province (grant no. BE2016646).

\section{References}

1. Stupp R, Hegi ME, Gilbert MR and Chakravarti A: Chemoradiotherapy in malignant glioma: Standard of care and future directions. J Clin Oncol 25: 4127-4136, 2007. 
2. Sanai N, Alvarez-Buylla A and Berger MS: Neural stem cells and the origin of gliomas. N Engl J Med 353: 811-822, 2005.

3. Wen PY and Kesari S: Malignant gliomas in adults. N Engl J Med 359: 492-507, 2008.

4. Roberts PJ and Der CJ: Targeting the Raf-MEK-ERK mitogen-activated protein kinase cascade for the treatment of cancer. Oncogene 26: 3291-3310, 2007.

5. Pandey V, Bhaskara VK and Babu PP: Implications of mitogen-activated protein kinase signaling in glioma. J Neurosei Res 94: 114-127, 2016.

6. Dong Chen, Waters SB, Holt KH and Pessin JE: SOS phosphorylation and disassociation of the Grb2-SOS complex by the ERK and JNK signaling pathways. J Biol Chem 271: 6328-6332, 1996.

7. Bhaskara VK, Panigrahi M, Challa S and Babu PP: Comparative status of activated ERK1/2 and PARP cleavage in human gliomas. Neuropathology 25: 48-53, 2005.

8. Xie H, Xue YX, Liu LB, Wang P, Liu YH and Ying HQ Expressions of matrix metalloproteinase-7 and matrix metalloproteinase-14 associated with the activation of extracellular signal-regulated kinase1/2 in human brain gliomas of different pathological grades. Med Oncol 28 (Suppl 1): S433-S438, 2011.

9. Lundberg JO, Gladwin MT and Weitzberg E: Strategies to increase nitric oxide signalling in cardiovascular disease. Nat Rev Drug Discov 14: 623-641, 2015.

10. Nakamura T and Lipton SA: Protein S-nitrosylation as a therapeutic target for neurodegenerative diseases. Trends Pharmacol Sci 37: 73-84, 2016

11. Wang Z: Protein S-nitrosylation and cancer. Cancer Lett 320 123-129, 2012

12. Feng X, Sun T, Bei Y, Ding S, Zheng W, Lu Y and Shen P: S-nitrosylation of ERK inhibits ERK phosphorylation and induces apoptosis. Sci Rep 3: 1814, 2013.

13. Shen A, Gao S, Ben Z, Wang H, Jia J, Tao T, Niu S, Li X and Cheng C: Identification and potential role of PSD-95 in Schwann cells. Neurol Sci 29: 321-330, 2008.

14. Louis DN, Ohgaki H, Wiestler OD and Cavenee WK (eds): WHO Classification of Tumours of the Central Nervous System. IARC WHO Classification of Tumours, Lyon, 2016.

15. Kurimoto M, Endo S, Hirashima Y, Hamada H, Ogiichi T and Takaku A: Growth inhibition and radiosensitization of cultured glioma cells by nitric oxide generating agents. J Neurooncol 42 : 35-44, 1999.

16. Stamler JS, Toone EJ, Lipton SA and Sucher NJ: (S)NO signals: Translocation, regulation, and a consensus motif. Neuron 18: 691-696, 1997.

17. Weyerbrock A, Baumer B and Papazoglou A: Growth inhibition and chemosensitization of exogenous nitric oxide released from NONOates in glioma cells in vitro. J Neurosurg 110: 128-136, 2009 .
18. Maejima Y, Adachi S, Morikawa K, Ito $\mathrm{H}$ and Isobe M: Nitric oxide inhibits myocardial apoptosis by preventing caspase-3 activity via S-nitrosylation. J Mol Cell Cardiol 38: 163-174, 2005.

19. Lechner M, Lirk P and Rieder J: Inducible nitric oxide synthase (iNOS) in tumor biology: The two sides of the same coin. Semin Cancer Biol 15: 277-289, 2005.

20. Lancaster JR Jr and Xie K: Tumors face NO problems? Cancer Res 66: 6459-6462, 2006.

21. Wang Y, Chen C, Loake GJ and Chu C: Nitric oxide: Promoter or suppressor of programmed cell death? Protein Cell 1: 133-142, 2010.

22. Azad N, Vallyathan V, Wang L, Tantishaiyakul V, Stehlik C, Leonard SS and Rojanasakul Y: S-nitrosylation of Bcl-2 inhibits its ubiquitin-proteasomal degradation. A novel antiapoptotic mechanism that suppresses apoptosis. J Biol Chem 281:34124-34134, 2006.

23. Chanvorachote $P$, Nimmannit U, Stehlik C, Wang L, Jiang BH, Ongpipatanakul B and Rojanasakul Y: Nitric oxide regulates cell sensitivity to cisplatin-induced apoptosis through S-nitrosylation and inhibition of Bcl-2 ubiquitination. Cancer Res 66: 6353-6360, 2006.

24. Leon-Bollotte L, Subramaniam S, Cauvard O, Plenchette-Colas S, Paul C, Godard C, Martinez-Ruiz A, Legembre P, Jeannin JF, Bettaieb A: S-nitrosylation of the death receptor fas promotes fas ligand-mediated apoptosis in cancer cells. Gastroenterology 140: 2009-2018, 2018.e2001-2004, 2011.

25. Sebolt-Leopold JS and Herrera R: Targeting the mitogen-activated protein kinase cascade to treat cancer. Nat Rev Cancer 4: 937-947, 2004.

26. Murillo-Carretero M, Torroglosa A, Castro C, Villalobo A and Estrada C: S-nitrosylation of the epidermal growth factor receptor: A regulatory mechanism of receptor tyrosine kinase activity. Free Radic Biol Med 46: 471-479, 2009.

27. Kaliyaperumal K, Sharma AK, McDonald DG, Dhindsa JS, Yount C, Singh AK, Won JS and Singh I: S-nitrosoglutathione-mediated STAT3 regulation in efficacy of radiotherapy and cisplatin therapy in head and neck squamous cell carcinoma. Redox Biol 6: 41-50, 2015.

28. Chattopadhyay M, Goswami S, Rodes DB, Kodela R, Velazquez CA, Boring D, Crowell JA and Kashfi K: NO-releasing NSAIDs suppress NF- $\kappa$ B signaling in vitro and in vivo through S-nitrosylation. Cancer Lett 298: 204-211, 2010.

29. Szabo C: Gasotransmitters in cancer: From pathophysiology to experimental therapy. Nat Rev Drug Discov 15: 185-203, 2016.

(i) $\odot$ This work is licensed under a Creative Commons Attribution-NonCommercial-NoDerivatives 4.0 International (CC BY-NC-ND 4.0) License. 